\title{
Using Series to Study the Relationship between Interval Continuity and Conductance
}

\author{
Jiandong $\mathrm{Ma}$
}

School of Information \& Communication Engineering, University of Electronic Science and Technology of China, No.2006, Xiyuan Ave, West Hi-Tech Zone, Chengdu, Sichuan, P.R. China

majiandongg@126.com

Keywords: Continuity; Conductive; Range; Weierstrass function item level

\begin{abstract}
The relation between continuity of function and conductivity is already clear in mathematical analysis. For example, if a function is continuous at some point, it may or may not be derivative at this point. However, when the function is continuous in the range, it is possible that the function cannot be induced in the interval but there is not enough counter-example or proof. Therefore, this paper takes the interval as the research object. By using the related properties of the series of Weierstrass functions, this paper gives a series of continuous but not all-pervading functions in the interval, and carries out theoretical proof, image research and analogy construction, further deepens the understanding of regional function continuity and derivability.
\end{abstract}

\section{Definition of Continuous and Definable Functions}

Continuity of Functions. Suppose the function $f(x)$ is defined at a certain neighboring region of the point $x_{0}$, if $\lim _{x \rightarrow x_{0}} f(x)=f\left(x_{0}\right)$, the function is continuous at $x_{0}$ and $x_{0}$ is the continuity point of the function.

Derivability of Functions. (1) Suppose $f(x)$ is defined at $x_{0}$ or the neighboring area, when a is getting closer to 0 , if the limit of $\lim _{a \rightarrow 0} \frac{f\left(x_{0}+a\right)-f\left(x_{0}\right)}{a}$ exists, then $f(x)$ is derivable at $x_{0}$. (2) If any point $(\mathrm{m}, \mathrm{f}(\mathrm{m}))$ within the range $(\mathrm{a}, \mathrm{b})$ is derivable, then $\mathrm{f}(\mathrm{x})$ is derivable at $(\mathrm{a}, \mathrm{b})$.

\section{Relationship between Continuity and Derivability of Functions at a Certain Point}

Suppose the function $y=f(x)$ is derivable at point $x_{0}$, then $y=f(x)$ must be continuous at point $x_{0}$. (1)

Prove: because the known condition $\lim _{\Delta x \rightarrow 0} \frac{\Delta y}{\Delta x}=f^{\prime}\left(x_{0}\right)$ exists, then

$$
\begin{aligned}
& \frac{\Delta \mathrm{y}}{\Delta x}=f^{\prime}\left(x_{0}\right)+\alpha \quad(\text { when } \Delta \mathrm{x} \rightarrow 0, \alpha \text { is infinitely small), } \\
& \Delta y=f^{\prime}\left(x_{0}\right) \Delta x+\alpha \Delta x=f^{\prime}\left(x_{0}\right) \Delta x+o(\Delta x), \\
& \text { so } \lim _{\Delta x \rightarrow 0} \Delta y=0 \\
& \text { and the function } y=f(x) \text { continues at point } x_{0} .
\end{aligned}
$$

Suppose the function $y=f(x)$ is continuous at the point $x_{0}$, then $y=f(x)$ may be derivable or not at the point $x_{0}$.

Prove: The function is continuous at a certain point, then the proof that this formula is derivable at this point can be omitted.

Therefore, prove the follow that there is no possibility for the function to be continuous but non-derivable at a certain point. 
Take the function $f(x)=|x|$ as an example. This function is continuous everywhere, but is non-derivable at the point $x_{0}$ (The derivative at this point is not the same). Therefore, when a function is continuous at a certain point, it may not be derivable.

\section{Relationship between Continuity and Derivability in the Function Range}

Suppose the function $y=f(x)$ is derivable at a certain range, then $y=f(x)$ must be continuous at this range.

Prove: Suppose the function $y=f(x)$ is derivable at ( $\mathrm{a}, \mathrm{b})$.

Then based on the results of the proof in (1), the generalization and continuity of each point in the interval can be extended to the continuity and derivability of the interval. The proof is omitted.

Therefore, $y=f(x)$ must be continuous at this derivable range.

Suppose the function $y=f(x)$ is continuous within a certain range, then $y=f(x)$ may be derivable or not within this range.

Prove: The proof that the function may be continuously derivable within a certain range is omitted.

Question: We were having trouble trying to prove that the function may not be continuous in a certain range. Most people will intuitively think that a continuous function must be nearly derivable. Many early mathematicians, including Gauss, had assumed that the non-conductive parts of continuous functions were derivable [1]. This may be due to the fact that it is difficult to visualize a continuous but non-derivative function that is not infinite. Just as we draw an image of a function, we draw a graph of consecutive rules.

Until 1872, Wellsstraith constructed the Weierstrass function using series knowledge, with astonishingly continuous, non-conductive properties, giving the answers to the above questions. In 1930, Van der Waerde proposed a more straightforward, continuous, non-conductive function that is also defined in terms of series tools.

Use the Van der Waerde function to prove that the function interval may not be non-derivable everywhere [2]

Prove: form of Van der Waerde function: $f(x)=\sum_{n=0}^{\infty} \frac{\varphi\left(10^{n} x\right)}{10^{n}}$

In which, $\varphi(\mathrm{x})$ refers to the distance between $\mathrm{x}$ and the nearest integer. For example, when $\varphi(1.26)=0.26, \varphi(3.67)=0.33$.

Obviously, $\varphi(\mathrm{x}) \leq \frac{1}{2}$, and it is a continuous function with the cycle as 1 . Besides, when $x, y \in\left[k, k+\frac{1}{2}\right]$ or $\left[\frac{k+1}{2}, k+1\right],(\mathrm{k} \in \mathrm{Z}),|\varphi(x)-\varphi(y)|=|x-y|$.

Prove the regional derivability of the function, it can be known from $\left|\frac{\varphi\left(10^{\mathrm{n}} \mathrm{x}\right)}{10^{n}}\right| \leq \frac{1}{2 \cdot 10^{n}}$ and the convergence of $\sum_{n=0}^{\infty} \frac{1}{2 \cdot 10^{n}}$ as well as the Weierstrass principle that the above function items are about the consistent convergence of $x \in R$. According to the theorem 2.3[3], it can be known that the original function is continuous at $\mathrm{R}$.

Prove the regional non-derivability of the function: select a point $\mathrm{x}$ at the function $f(x)_{\mathrm{at}}$ random. Due to the cycle of $\varphi(\mathrm{x})$, set $0 \leq \mathrm{x}<1$. Express $\mathrm{x}$ as infinite decimal $x=0 . a_{1} a_{2} \ldots \ldots a_{n} \ldots \ldots$ If $\mathrm{x}$ is a finite decimal, add countless 0 after it. And then take

$$
h_{m}=10^{-\mathrm{m}} \quad \text { when } a_{m}=0,1,2,3,5,6,7,8
$$




$$
=-10^{-\mathrm{m}} \quad \text { when } a_{m}=4,9,
$$

If $\mathrm{x}=0.23490 \ldots$, then take $h_{1}=10^{-1}, h_{2}=10^{-2}, h_{3}=-10^{-3}, h_{4}=-10^{-4}, h_{5}=10^{-5} \quad$ Obviously, when $m \rightarrow \infty, \quad h_{m} \rightarrow 0$. Therefore, only when the limit $\lim _{m \rightarrow \infty} \frac{f\left(x+h_{m}\right)-f(x)}{h_{m}}$ is proven to be

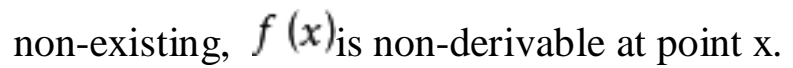

$$
\begin{aligned}
& \frac{f\left(x+h_{m}\right)-f(x)}{h_{m}}=\sum_{n=0}^{\infty} \frac{\varphi\left(10^{n}\left(x+h_{m}\right)\right)-\varphi\left(10^{n} x\right)}{10^{n} h_{m}}= \\
& \sum_{n=0}^{m-1} \frac{\varphi\left(10^{n}\left(x+h_{m}\right)\right)-\varphi\left(10^{n} x\right)}{10^{n} h_{m}}+\sum_{n=m}^{\infty} \frac{\varphi\left(10^{n}\left(x+h_{m}\right)\right)-\varphi\left(10^{n} x\right)}{10^{n} h_{m}}
\end{aligned}
$$

When $\mathrm{n} \geq \mathrm{m}, \varphi\left(10^{\mathrm{n}}\left(x+h_{m}\right)\right)=\varphi\left(10^{n} x \pm 10^{n-m}\right)=\varphi\left(10^{\mathrm{n}} x\right)$, so

$$
\begin{aligned}
& \sum_{n=m}^{\infty} \frac{\varphi\left(10^{n}\left(x+h_{m}\right)\right)-\varphi\left(10^{n} x\right)}{10^{n} h_{m}}=0 \\
& \frac{f\left(x+h_{m}\right)-f(x)}{h_{m}}=\sum_{n=0}^{\mathrm{m}-1} \frac{\varphi\left(10^{n}\left(x+h_{m}\right)\right)-\varphi\left(10^{n} x\right)}{10^{n} h_{m}}
\end{aligned}
$$

When $\mathrm{n}=0,1,2, \ldots, \mathrm{m}-1$, in the expression of $10^{\mathrm{n}} \mathrm{x}$, the location of $a_{m}$ is the $\mathrm{m}-\mathrm{n}$ decimal, $10^{n} x=a_{1} a_{2} \ldots a_{\mathrm{n}} \cdot a_{n+1} \ldots a_{m} \ldots$, $10^{n}\left(x+h_{m}\right)=a_{1} a_{2} \ldots a_{\mathrm{n}} \cdot a_{n+1} \ldots\left(a_{m} \pm 1\right) \ldots$,

Due to the selected $h_{m}$, it can be known that $10^{n}\left(x+h_{m}\right)$ and $10^{n} x$ both belong to $\left[k, k+\frac{1}{2}\right]$ or $\left[\frac{k+1}{2}, k+1\right]$, so $\varphi\left(10^{n}\left(x+h_{m}\right)\right)-\varphi\left(10^{n} x\right)= \pm 10^{n} h_{m}$

Put (3) into (2), and it can be known that $\frac{f\left(x+h_{m}\right)-f(x)}{h_{m}}=\sum_{n=0}^{\mathrm{m}-1} \pm 1$

The right end of the equation must be even, and its parity and $m$ are known. Thus, it can be known that the limit $\lim _{m \rightarrow \infty} \frac{f\left(x+h_{m}\right)-f(x)}{h_{m}}$ does not exist. That is to say, $f(x)_{\text {is non-derivable at }}$ any point $x$. In this way, A contiguous, but ubiquitous, function counterexample was constructed by a function-level series tool.

\section{Continuous but Non-conductive Function Series}

Basic Principles of Van der Waerde Function. Looking back at the Van der Waerde function, we can conclude the rationale for constructing a series of function-term series that are nonconductive at all times. Its core is to use the bounded periodic function $\varphi_{\text {as a function of left and right }}$ derivative of the same time to ensure that the continuity of the function itself is not destroyed.

Of course, it is undeniable that the first example of a ubiquitous continuous function is proposed by Wilstras with the construction of the vibration curve $\mathrm{y}=\cos (\pi \mathrm{x}): \sum_{n=0}^{\infty} b^{n} \cos \left(a^{n} \pi x\right)$. VAn der Waerde improves the vibration curve to a polyline $\varphi$ ( $\mathrm{x}$ ) according to the example proposed by our predecessors, $x \in[-1,1]$ and the successfully construct a more simple and similar function series.

In addition, in the proof of Van der Waerde's opposite examples, the selection of symbol $h_{m}$ is crucial. When $a_{m}=4$ or 9 , it is negative, and when $a_{m}$ is other numbers, it is positive. This selection 
of symbol ensures that when $\mathrm{n}=0,1,2, \ldots \ldots, \mathrm{m}-1,10^{n}\left(x+h_{m}\right)$ and $10^{n} x$ both belong to $\left[k, k+\frac{1}{2}\right]$ or $\left[\frac{k+1}{2}, k+1\right]$, so there is $\frac{f\left(x+h_{m}\right)-f(x)}{h_{m}}=\sum_{n=0}^{\mathrm{m}-1} \frac{\varphi\left(10^{n}\left(x+h_{m}\right)\right)-\varphi\left(10^{n} x\right)}{10^{n} h_{m}}=\sum_{n=0}^{m-1} \pm 1$

Construct Similar and Continuous Non-Derivative Function Terms. Set the function $f(x)=\sum_{n=1}^{\infty} f_{n}(x)=\sum_{n=1}^{\infty} \frac{f_{1}\left(4^{n-1}\right)}{4^{n-1}}, x \in R$

In which $f_{1}(x)=|x| \leq \frac{1}{2}$

$$
f_{1}(x+n) \quad \text { Other cases }
$$

Such as $f_{n}(x)=4^{-n+1} f_{1}\left(4^{n-1} x\right), n=2,3, \ldots \ldots$

Because $\left|f_{n}(x)\right| \leq \frac{1}{2} \cdot 4^{-n+1}$, according to Weierstrass principle, it can be known that this level converges all the time at R. According to theorem 2.3, then the original function continues everywhere at $\mathrm{R}$.

Take any point $\mathrm{x}$ at $\mathrm{R}$, for any natural number $\mathrm{n}$, take $h_{n}=4^{-n-1}$ or $h_{n}=-4^{-n-1}$ to make

$$
\begin{gathered}
\left|f\left(x+h_{n}\right)-f_{n}(x)\right|=\left|h_{n}\right| \\
\text { So }\left|f_{\mathrm{m}}\left(x+h_{n}\right)-f_{m}(x)\right|= \\
0 \quad\left|h_{n}\right| \quad \mathrm{m} \leq \mathrm{n}
\end{gathered}
$$

Therefore, $\frac{f\left(x+h_{n}\right)-f(x)}{h_{n}}$ is an integer.

So that $\lim _{n \rightarrow \infty} \frac{f\left(x+h_{n}\right)-f(x)}{h_{n}}$ does not exist. That is, for any real number $\mathrm{x}, f^{\prime}(x)$ does not exist, i.e., $f(x)$ is non-derivative everywhere.

Of course, there are many similar series of function items. For example, in 1952, Bush proposed the simplest and most elementary asymptotically continuous function, using the definition of fractional expansion of real numbers [5]; defining an infinite jagged function in the interval [4]; According to Weierstrass function to change its sign, limit conditions, a series of extended domain function [5]. These series of functions have achieved the continuous non-conductive nature everywhere, and we will not repeat them here.

Image Fractal Properties of the Weierstrass Function. Although we have already deduced theoretically that these functions have properties that cannot be continuously and uniformly everywhere in the interval, we still want to know how the function images in the coordinate system are displayed. What are the special properties? Using mathematical software, it is not hard to draw the Weierstrass function (Fig.1) [6] and the infinite jagged function mentioned above [5] (Fig. 2):

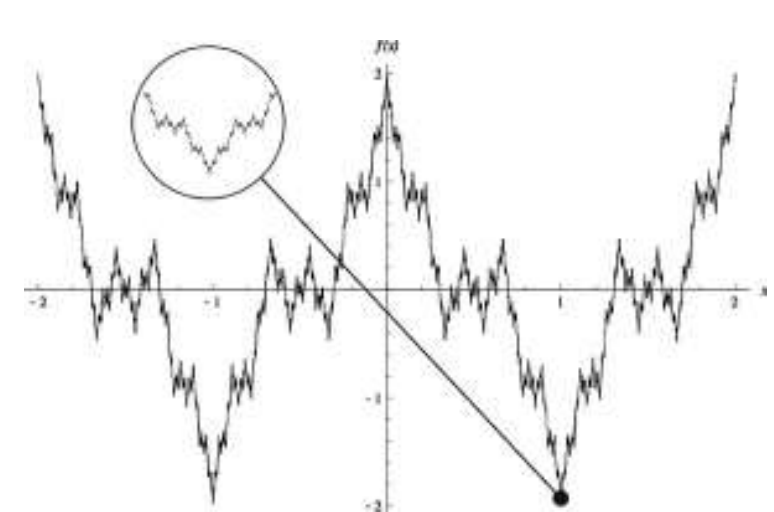

Figure.1

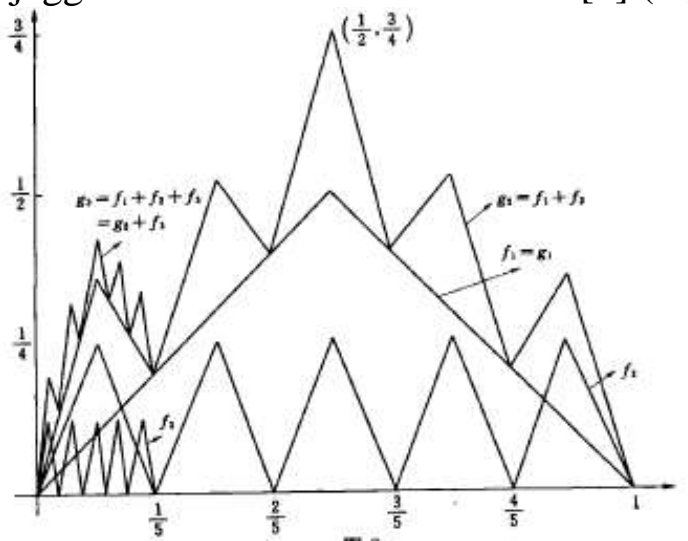

Figure. 2 
As can be seen, the local and global aspects of such functions are similar in some way, that is, their parts are "self-similar" to their entirety. Therefore, it is not difficult to understand the special relationship between the continuity and the guiding of the Weierstrass function itself. Ordinary function will be infinitely magnified, and the function image will always be approximated as a straight line. However, if the Weierstrass function is infinitely enlarged, the function image will never converge to a straight line but will be fractalized indefinitely into its original shape. So, we can see that the Weierstrass function is continuous but is non-conductive.

The fractal geometry was first established by Benoit B. Mandelbrot in 1975, and the Weierstrass function that preceded it was precisely one of the classical models of fractal geometry.

\section{Conclusion}

Recalling the history of mathematics that argues for the nonconductivity of continuum everywhere, and the argument of the continuity of function continuity, we cannot help but feel that constructing counter examples plays an equally important role in giving proofs. Counterexamples sometimes directly promote the formation and development of new concepts, new theorems and new theories in mathematics. As Olmsted argues: "Mathematics consists of two major categories - proofs and counter-examples, and mathematical discoveries are directed toward two main goals - propositions and counter-examples." [7]

Finally, by using the continuity and adaptability of the inquiry function as an example, it is found that the missing of the counterfeit example of "the function can be guided everywhere in a certain interval". Thus, after access to relevant information, it is found that Weierstrass function series have ubiquitous non-conductive properties everywhere. Through the theoretical proofs, theory discussion, image analysis and analogy construction of series functions of similar nature, closely linked the series knowledge, the function knowledge and the periodic vibration, we've learned the construction principle of the correlation function and mastered the image of the fractal function, further sublimation of the continuity of function and the connection between the understanding of guidance.

\section{Acknowledgements}

This project was funded by the Humanities and Social Sciences Planning Foundation of the Ministry of Education (No. 10YJAZH056), Shandong Province Soft Science Research Projects (No. 2009RKB439).

\section{References}

[1] Wang Xiaoming. Fractal Properties of Continuous and Differentiable Functions. (Doctoral dissertation, Jiangsu University, China, 2006), p.2. (In Chinese).

[2] Chen Ji Xiu, and Qiu Wei Yuan: A Counter-example in Mathematical Analysis Courses Functions that Cannot be Followed Everywhere. Advanced Mathematics Research, Vol. 9 (2006) No.7, p.2.

[3] Ma Zhi'en and Wang Miansen: "Some Experience with Writing Teaching Materials for the $21^{\text {st }}$ Century". Chinese University Teaching, Vol.9 (1999)p.36.

[4] Li Yuanhua, and Yu Mao Di: "A simple Wesstraat Function". College Mathematics, Vol.22 (2006) No.2, p118.

[5] Zhongyuan Tao, Yuan Li, and Deng Xin: "Several Methods to Construct Non-conductible Continuous Functions". Journal of Yunyang Teachers College, Vol.23 (2003) No.6, p10.

[6] Information on https://baike.baidu.com/item/ Wells Stras function /4191720?fr=aladdin

[7] Ma Changwei: "Analysis on the Construction of Counterexamples in Mathematical Analysis". Journal of Aba Teachers College, Vol.2 (2002) p.103. 